\title{
Transformation Omnithermotics: Simultaneous Manipulation of Three Basic Modes of Heat Transfer
}

Liujun $\mathrm{Xu}^{*}$, ShuaiYang, Gaole Dai and Jiping Huang*

Conduction, convection, and radiation are three basic modes of heat transfer which often exist together. However, it is up to now a challenge to simultaneously manipulate them within the framework of transformation theory because they possess diverse properties with different mechanisms. To solve this problem, we develop a transformation theory to control them simultaneously (herein called transformation omnithermotics). With the present theory, we further design three devices including omnithermal cloaking, concentrating, and rotating as model applications. Finite-element simulations and experimental suggestions are also provided to confirm these applications. This work not only unifies the three basic modes of heat transfer within the theoretical framework of transformation omnithermotics, but also provides novel hints and potential applications to thermal management.

Keywords: Transformation omnithermotics; Omnithermal metamaterials; Heat transfer

Received 18 January 2020, Accepted 12 February 2020

DOI: $10.30919 /$ esee 8 c372

\section{Introduction}

It is known that heat energy can be transferred by three basic modes including conduction, convection, and radiation. They have diverse properties with different mechanisms, but often appear simultaneously. For example, solar vapour generation ${ }^{1-4}$ is a typical system which contains the three basic modes of heat transfer: The utilization of solar energy is related to radiation, the evaporation of seawater is associated with convection, and conduction exitsin almost each process. Another typical example is the heat transfer in aerogels ${ }^{5}$ where the basic three modes of heat transfer should also be taken into consideration. On all accounts, the underlying physics is the simultaneous manipulation of conduction, convection, and radiation, which is of particular significance for thermal management.

Transformation theory, as a general and powerful methodology, has already been extended from wave systems (e.g., electromagnetic propagation ${ }^{6}$ ) to diffusion systems (e.g., heat conduction ${ }^{7,8}$ ), thus yielding the rapid development of thermal metamaterials or metadevices, ${ }^{9}$ such as cloaks, ${ }^{10-18}$ concentrators, ${ }^{10,18-21}$ rotators, ${ }^{10,18}$ camouflaging, ${ }^{22-28}$ and guiding. ${ }^{29-31}$ However, these thermal metamaterials take only conductive effects into consideration. Recently, some studies take convective effects ${ }^{32-35}$ into account to promote new applications of thermal metamaterials.

However, there does not exist a transformation theory to deal with conduction, convection, and radiation simultaneously. Owing to their distinctly different mechanisms, they seem difficult to be unified within the same framework of transformation theory. Since they often appear simultaneously, it becomes important and urgent to develop a transformation theory to deal with them simultaneously.

For this purpose, we propose the theory of transformation omnithermotics by considering the three basic modes of heat transfer at the same time. This theory further helps us design three devices including omnithermal cloaking, concentrating, and rotating as model applications. In this regard, cloaks can protect objects in the center from being detected, concentrators can enhance local heat flux, and rotators can control the direction of local heat flux. These three devices are confirmed

Department of Physics, State Key Laboratory of Surface Physics, and Key Laboratory of Micro and Nano Photonic Structures (MOE), Fudan University, Shanghai 200438, China

*E-mail: 13307110076@fudan.edu.cn;jphuang@fudan.edu.cn 
by finite-element simulations, and exhibit good performance in both steady and transient states. We also resort to porous media to control flow velocity to ensure feasibility. In what follows, let us begin by presenting the theory.

\section{Theory of Transformation Omnithermotics}

Fluids or gases are indispensable to the existence of convective flux. In spite of different forms, they follow the same equation in terms of heat transfer, so we just discuss fluids without the effects of solubility for brevity. We further suppose that fluids are incompressible and laminar with a low speed, so we can neglect the momentum conservation of fluids when handling thermal convection. Therefore, the transient process of heat transfer with conduction, convection, and radiation is dominated by

$$
\begin{gathered}
\rho_{f} C_{f} \partial T / \partial t+\nabla \cdot\left(-\boldsymbol{\kappa}_{f} \cdot \nabla T+\rho_{f} C_{f} \boldsymbol{v}_{f} T-\boldsymbol{\alpha}_{f} T^{3} \cdot \nabla T\right)=0, \\
\partial \rho_{f} / \partial t+\nabla \cdot\left(\rho_{f} \boldsymbol{v}_{f}\right)=0,
\end{gathered}
$$

where $\rho_{f}, C_{f}, \boldsymbol{\kappa}_{f}$, and $\boldsymbol{v}_{f}$ are the density, heat capacity, thermal conductivity, and velocity of fluids, respectively. $\boldsymbol{\alpha}_{f}\left(=16 \boldsymbol{\beta}_{f}^{-1} \boldsymbol{n}_{f}^{2} \sigma / 3\right)$ can be treated as radiative coefficient where $\boldsymbol{\beta}_{f}, \quad \boldsymbol{n}_{f}$, and $\sigma\left(=5.67 \times 10^{-8} \mathrm{Wm}^{-2} \mathrm{~K}^{-4}\right)$ are the Rosseland mean attenuation coefficient, relative refractive index, and the Stefan-Boltzmann constant, respectively. $T$ and $t$ denote temperature and time, respectively. Physically speaking, the two equations in Eq. (1) describe the conservations of heat flux and mass flux, respectively. Conductive flux $\boldsymbol{J}_{1}$ is determined by the Fourier law $\boldsymbol{J}_{1}=$ $-\boldsymbol{\kappa}_{f} \cdot \nabla T$; convective flux $\boldsymbol{J}_{2}$ is given by $\boldsymbol{J}_{2}=\rho_{f} C_{f} \boldsymbol{v}_{f} T ;{ }^{35}$ radiative flux $J_{3}$ is dealt with the Rosseland diffusion approximation $\boldsymbol{J}_{3}=-\boldsymbol{\alpha}_{f} T^{3} \cdot \nabla T ;{ }^{36}$ and total flux $\boldsymbol{J}_{T}$ is the summation of conductive, convective, and radiative fluxes, namely $\boldsymbol{J}_{\mathrm{T}}=\boldsymbol{J}_{1}+\boldsymbol{J}_{2}+\boldsymbol{J}_{3}$.

Equation (1) can keep its form-invariance after a space transformation from a curvilinear space $S$ to a physical space $S^{\prime}$, which is determined by the Jacobian transformation matrix $\Lambda$. To prove this, we write down the component form of Eq. (1). In a curvilinear space with a set of contravariant basis $\left(\boldsymbol{g}^{i}, \boldsymbol{g}^{j}, \boldsymbol{g}^{k}\right)$ and corresponding contravariant components $\left(s^{i}, s^{j}, s^{k}\right)$, Eq. (1) can be rewritten as

$$
\begin{gathered}
\sqrt{g} \rho_{f} C_{f} \partial_{t} T+\partial_{i}\left(\sqrt{g}\left(-\kappa_{f}^{i j} \partial_{j} T+\rho_{f} C_{f} v_{f}^{i} T-\alpha_{f}^{i j} T^{3} \partial_{j} T\right)\right)=0, \\
\sqrt{g} \partial_{t} \rho_{f}+\partial_{i}\left(\sqrt{g} \rho_{f} v_{f}^{i}\right)=0,
\end{gathered}
$$

where $g$ is the determinant of the matrix $\boldsymbol{g}_{i} \cdot \boldsymbol{g}_{i}$, and $\left(\boldsymbol{g}_{i}, \boldsymbol{g}_{j}, \boldsymbol{g}_{k}\right)$ is a set of covariant basis. Eq. (2) is expressed in the curvilinear space, which should be rewritten in the physical space with the Cartesian coordinates $\left(s^{i^{\prime}}, s^{j^{\prime}}, s^{k^{\prime}}\right)$,

$$
\begin{gathered}
\partial_{i^{\prime}} \frac{\partial s^{i^{\prime}}}{\partial s^{i}}\left(\sqrt{g}\left(-\kappa_{f}^{i j} \frac{\partial s^{j^{\prime}}}{\partial s^{j}} \partial_{j^{\prime}} C_{f} \partial_{t} T+\rho_{f} C_{f} v_{f}^{i} T-\alpha_{f}^{i j} T^{3} \frac{\partial s^{j^{\prime}}}{\partial s^{j}} \partial_{j^{\prime}} T\right)\right)=0 \\
\sqrt{g} \partial_{t} \rho_{f}+\partial_{i^{\prime}} \frac{\partial s^{i^{\prime}}}{\partial s^{i}}\left(\sqrt{g} \rho_{f} v_{f}^{i}\right)=0
\end{gathered}
$$

where $\partial s^{i^{\prime}} / \partial s^{i}$ and $\partial s^{j^{\prime}} / \partial s^{j}$ are just the components of the Jacobian transformation matrix $\Lambda$, and $\sqrt{g}=1 / \operatorname{det} \Lambda$. The essence of transformation theory is to turn space transformations into material transformations. For this purpose, we rewrite Eq. (3) as

$$
\begin{aligned}
& \frac{\rho_{f}}{\operatorname{det} \Lambda} C_{f} \partial_{t} T+ \\
& \partial_{i^{\prime}}\left(-\frac{\frac{\partial s^{i^{\prime}}}{\partial s^{i}} \kappa_{f}^{i j} \frac{\partial s^{j^{\prime}}}{\partial s^{j}}}{\operatorname{det} \Lambda} \partial_{j^{\prime}} T+\frac{\rho_{f}}{\operatorname{det} \Lambda} C_{f} \frac{\partial s^{i^{\prime}}}{\partial s^{i}} v_{f}^{i} T-\frac{\frac{\partial s^{i^{\prime}}}{\partial s^{i}} \alpha_{f}^{i j} \frac{\partial s^{j^{\prime}}}{\partial s^{j}}}{\operatorname{det} \Lambda} T^{3} \partial_{j^{\prime}} T\right)=0 \\
& \partial_{t} \frac{\rho_{f}}{\operatorname{det} \Lambda}+\partial_{i^{\prime}}\left(\frac{\rho_{f}}{\operatorname{det} \Lambda} \frac{\partial s^{i^{\prime}}}{\partial s^{i}} v_{f}^{i}\right)=0 .
\end{aligned}
$$

Therefore, we can obtain the transformation rules as

$$
\begin{gathered}
\rho_{f}^{\prime}=\rho_{f} / \operatorname{det} \Lambda, \\
C_{f}^{\prime}=C_{f}, \\
\boldsymbol{\kappa}_{f}^{\prime}=\Lambda \boldsymbol{\kappa}_{f} \Lambda^{\tau} / \operatorname{det} \Lambda, \\
\boldsymbol{v}_{f}^{\prime}=\Lambda \boldsymbol{v}_{f}, \\
\boldsymbol{\alpha}_{f}^{\prime}=\Lambda \boldsymbol{\alpha}_{f} \Lambda^{\tau} / \operatorname{det} \Lambda,
\end{gathered}
$$

where $\Lambda^{\tau}$ is the transpose of $\Lambda$. There are two parameters determining the radiative coefficient, namely the Rosseland mean attenuation coefficient and relative refractive index. For brevity, we do not transform relative refractive indexes when performing finite-element simulations, namely $\boldsymbol{n}_{f}^{\prime}=\boldsymbol{n}_{f}$. Therefore, the transformation of the Rosseland mean attenuation coefficient becomes $\boldsymbol{\beta}_{f}^{\prime}=\Lambda^{-\tau} \boldsymbol{\beta}_{f} \Lambda^{-1} \operatorname{det} \Lambda$.

\section{Results of Cloaking, Concentrating, and Rotating}

The space transformation of cloaking is

$$
\begin{gathered}
r^{\prime}=\frac{\left(R_{2}-R_{1}\right) r}{R_{2}}+R_{1}, \\
\theta^{\prime}=\theta,
\end{gathered}
$$

where $R_{1}$ and $R_{2}$ are the inner and outer radii, respectively.

The space transformation of concentrating is

$$
\begin{gathered}
r^{\prime}=\frac{R_{1} r}{R_{m}},\left(r<R_{m}\right) \\
r^{\prime}=\frac{\left(R_{1}-R_{m}\right) R_{2}+\left(R_{2}-R_{1}\right) r}{R_{2}-R_{m}},\left(R_{m}<r<R_{2}\right) \\
\theta^{\prime}=\theta,
\end{gathered}
$$

where $R_{m}$ is a medium radius determining the concentrating ratio.

The space transformation of rotating is 


$$
\begin{gathered}
r^{\prime}=r, \\
\theta^{\prime}=\theta+\theta_{0},\left(r<R_{1}\right) \\
\theta^{\prime}=\theta+\frac{\left(r-R_{2}\right) \theta_{0}}{\left(R_{1}-R_{2}\right)},\left(R_{1}<r<R_{2}\right)
\end{gathered}
$$

where $\theta_{0}$ is a rotation degree. Then, the Jacobian transformation matrix of Eqs. (6) - (8) can be calculated by

$$
\Lambda=\left(\begin{array}{cc}
\frac{\partial r^{\prime}}{\partial r} & \frac{\partial r^{\prime}}{r \partial \theta} \\
\frac{r^{\prime} \partial \theta^{\prime}}{\partial r} & \frac{r^{\prime} \partial \theta^{\prime}}{r \partial \theta}
\end{array}\right) .
$$

Since the dominant equation of heat transfer of omnithermotics [Eq. (1)] is comprehensive, COMSOL MULTIPHYSICS ${ }^{37}$ has not a ready-made template for us to proceed. Therefore, we should establish the model by ourselves in the interface with general partial differential equations. It is not difficult as long as we input the component form of Eq. (1).

We choose three cases to exhibit different influences of conduction, convection, and radiation. The first case has a temperature interval of $300-360 \mathrm{~K}$ with background velocity along $+x$ axis, where convection is dominant. The second case has a temperature interval of 300-1200 K with the same background velocity, where radiation starts playing a role. The third case has a temperature interval of 300-1200 K with background velocity along $-x$ axis, where convection weakens total flux because of the opposite direction of convective flux. It takes about 80,30 , and 50 mins for the three cases to reach steady states if the initial temperatures are set at $300 \mathrm{~K}$.

Then, we perform transient simulations of cloaking. The parameters are designed according to Eq. (6). Figs. 1(ad), 1(e-h), and 1(i-j) demonstrate the temperature evolutions over time under the three different cases, respectively. The boundary $r=R_{1}$ is set with insulated and no-flow conditions to avoid the problems resulting from the singular parameters derived from Eq. (6). In this way, the central white regions in Fig. 1 can be placed with any object. Meanwhile, the background isotherms are not distorted, thus confirming the cloaking performance.

As quantitative analyses, we also plot the conductive, convective, radiative, and total flux of the background in Figs. 1(d, h\&l). The distributions of heat fluxes are presented in Figs. 2(a-c), respectively. We observe that total flux $\boldsymbol{J}_{T}$ is a constant along $x$ axis, which is conservative as described by Eq. (1).

Concentrating is designed with Eq. (7). The isotherms in the center of each simulation in Fig. 3 are more intensive than those in the background, indicating larger heat fluxes. Therefore, the concentrating effect is achieved.

Rotating is designed with Eq. (8). The isotherms in the center of each simulation in Fig. 4 turn to be horizontal and high temperatures appear in the bottom, indicating the direction of heat fluxes is vertically upward. In other words, heat fluxes are successfully rotated $\pi / 2 \mathrm{rad}$.

\section{Manipulating Flow Velocity with Porous Media}

Although Eq. (1) can describe the transient process of heat transfer, it cannot describe the establishment of velocity fields. In other words, velocity fields are preestablished. Therefore, we have to transform flow velocity directly. It is completely right in mathematics, but extremely difficult in experiments. Therefore, we resort to porous media to control flow velocity and ensure feasibility.

We have supposed that fluids are incompressible and laminar with a low speed, so fluid flow in porous media can
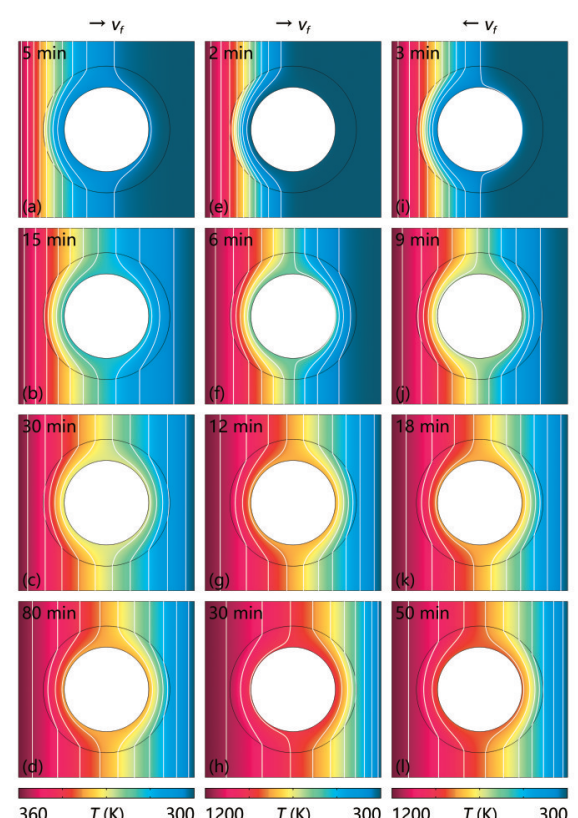

Fig. 1 Transient simulations of cloaking. Color surfaces denote temperature distributions, and white lines represent isotherms. The size of each simulation box is $0.1 \times 0.1 \mathrm{~m}^{2}, R_{1}=0.024 \mathrm{~m}$, and $R_{2}=0.036 \mathrm{~m}$, and the background fluids have parameters $\rho_{f}=1000 \mathrm{~kg} / \mathrm{m}^{3}$, $C_{f}=1000 \mathrm{~J} \mathrm{~kg}^{-1} \mathrm{~K}^{-1}, \kappa_{f}=1 \mathrm{~W} \mathrm{~m}^{-1} \mathrm{~K}^{-1}, v_{f}=10^{-5} \mathrm{~m} / \mathrm{s}, \beta_{f}=$ $100 \mathrm{~m}^{-1}$, and $n_{f}=1$ throughout the work. The transformation media and flow velocity are designed according to Eq. (6). (a)-(d), (e)-(h), and (i)-(1) show the temperature evolutions over time with three different cases, respectively. 
be described by the Darcy law $\boldsymbol{v}_{f}=-\boldsymbol{\eta}_{s} / \mu_{f} \cdot \nabla P,{ }^{38}$ where $\boldsymbol{\eta}$, is the permeability of porous media, $\mu_{f}$ is the dynamic viscosity of fluids, and $P$ denotes pressure. Then, Eq. (1) can be rewritten as

$$
\begin{gathered}
\rho_{m} C_{m} \partial T / \partial t+\nabla \cdot\left(-\boldsymbol{\kappa}_{m} \cdot \nabla T+\rho_{f} C_{f} \boldsymbol{v}_{f} T-\boldsymbol{\alpha}_{m} T^{3} \cdot \nabla T\right)=0, \\
\phi_{s} \partial \rho_{f} / \partial t+\nabla \cdot\left(\rho_{f} \boldsymbol{v}_{f}\right)=0, \\
\boldsymbol{v}_{f}=-\boldsymbol{\eta}_{s} / \mu_{f} \cdot \nabla P,
\end{gathered}
$$

where $\phi_{s}$ represents the porosity of porous media. $\rho_{m} C_{m}\left(=\left(1-\phi_{s}\right) \rho_{s} C_{s}+\phi_{s} \rho_{f} C_{f}\right), \quad \boldsymbol{\kappa}_{m}\left(=\left(1-\phi_{s}\right) \boldsymbol{\kappa}_{s}+\phi_{s} \boldsymbol{\kappa}_{f}\right)$, and $\boldsymbol{\alpha}_{m}\left(=\left(1-\phi_{s}\right) \boldsymbol{\alpha}_{s}+\phi_{s} \boldsymbol{\alpha}_{f}\right)$ are the mean values with respect to solids plus fluids, where $\rho_{s}, C_{s}, \boldsymbol{\kappa}_{s}$, and $\boldsymbol{\alpha}_{s}$ are the density, heat capacity, thermal conductivity, and radiative coefficient of solids, respectively. We can check that Eq. (10) also keeps its form-invariance after a space transformation, and the proof is similar to Eqs. (2-4). Thus, the transformation rules have forms as

$$
\begin{gathered}
\left(\rho_{m} C_{m}\right)^{\prime}=\rho_{m} C_{m} / \operatorname{det} \Lambda, \\
\boldsymbol{\kappa}_{m}^{\prime}=\Lambda \boldsymbol{\kappa}_{m} \Lambda^{\tau} / \operatorname{det} \Lambda, \\
\left(\rho_{f} C_{f}\right)^{\prime}=\rho_{f} C_{f}, \\
\boldsymbol{\alpha}_{m}^{\prime}=\Lambda \boldsymbol{\alpha}_{m} \Lambda^{\tau} / \operatorname{det} \Lambda, \\
\phi_{s}^{\prime}=\phi_{s} / \operatorname{det} \Lambda, \\
\boldsymbol{\eta}_{s}^{\prime}=\Lambda \boldsymbol{\eta}_{s} \Lambda^{\tau} / \operatorname{det} \Lambda, \\
\mu_{f}^{\prime}=\mu_{f} .
\end{gathered}
$$

Since the parameters of fluids are difficult to control, we do not transform them. Then, we can derive the transformation rules

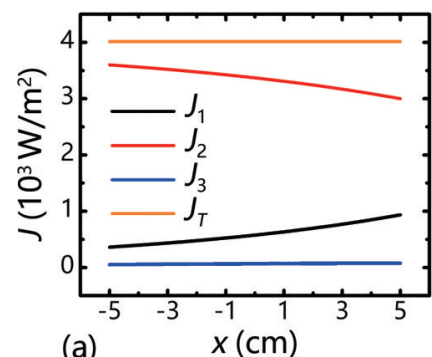

(a)

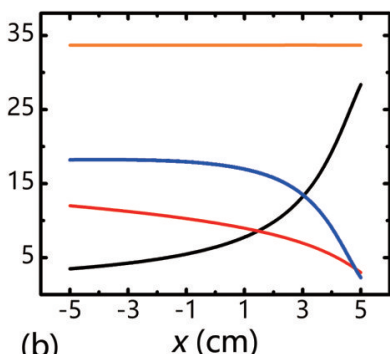

(b)

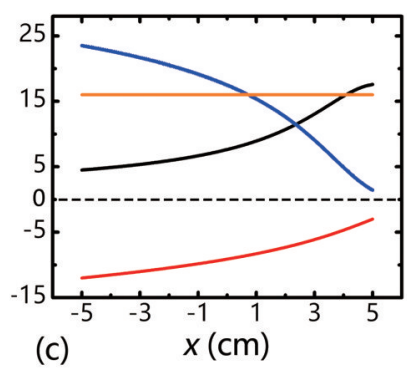

(c)

Fig. 2 Distributions of heat flux in background along $x$ axis. (a), (b), and (c) show the distributions of steady heat flux with the same boundary conditions applied in the first, second, and third columns in Fig. 2, respectively.

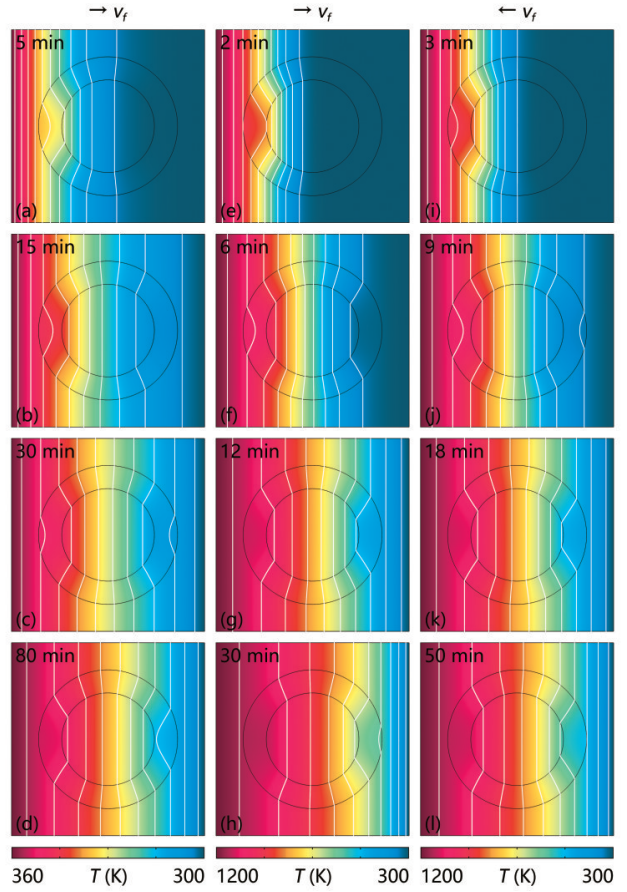

Fig. 3 Transient simulations of concentrating. The background parameters and boundary conditions are the same as those for Fig. 2. The transformation media and flow velocity are designed according to Eq. (7) where $R_{m}=0.032 \mathrm{~m}$.
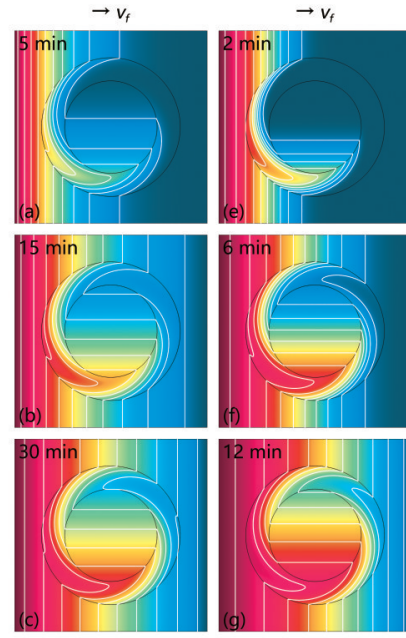
$\leftarrow v_{f}$
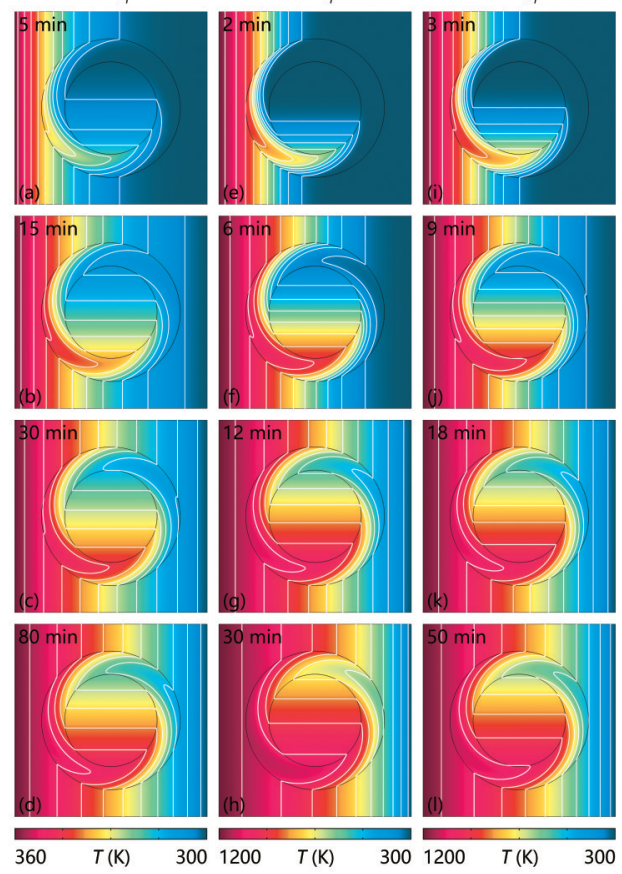

Fig. 4 Transient simulations of rotating. The background parameters and boundary conditions are the same as those for Fig. 2. The transformation media and flow velocity are designed according to Eq. (8) where $\theta_{0}=\pi / 2$. 
of solids as $\left(\rho_{s} C_{s}\right)^{\prime}=\left(1-\phi_{s}\right) \rho_{s} C_{s} /\left(\operatorname{det} \Lambda-\phi_{s}\right), \quad \boldsymbol{\kappa}_{s}^{\prime}=$ $\left(\Lambda \boldsymbol{\kappa}_{m} \Lambda^{\tau}-\phi_{s} \boldsymbol{\kappa}_{f}\right) /\left(\operatorname{det} \Lambda-\phi_{s}\right), \quad$ and $\boldsymbol{\alpha}_{s}^{\prime}=$ $\left(\Lambda \boldsymbol{\alpha}_{m} \Lambda^{\tau}-\phi_{s} \boldsymbol{\alpha}_{f}\right) /\left(\operatorname{det} \Lambda-\phi_{s}\right)$. In spite of the complex form of Eq. (11), its essence is the same as Eq. (5) except for the control of flow velocity. In Eq. (5), we directly transform flow velocity, whereas in Eq. (11), flow velocity is controlled by transforming the permeability of porous media. Meanwhile, the introduction of porous media can avoid directly transforming fluid parameters, which is more feasible.

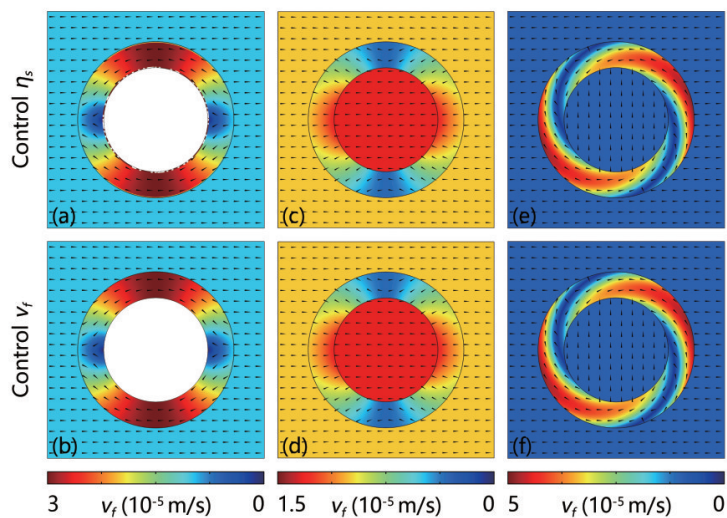

Fig. 5 Manipulating flow velocity with porous media. Color surfaces denote velocity distributions. (a), (c), and (e) are the velocity distributions obtained from transforming the permeability of porous media. (b), (d), and (f) are the velocity distributions obtained from directly transforming flow velocity. The boundary conditions of the first row are 1000 and $0 \mathrm{~Pa}$ for the left and right boundaries, and no-flow conditions for the upper and lower boundaries. The permeability of porous media in the background is $10^{-12} \mathrm{~m}^{2}$ (which is common in nature), and the dynamic viscosity of fluids is $0.001 \mathrm{~Pa} \mathrm{~s}$.

We also perform finite-element simulations to confirm these statements. The first row in Fig. 5 presents the results with transforming the permeability of porous media as $\boldsymbol{\eta}_{s}^{\prime}=$ $\Lambda \boldsymbol{\eta}_{s} \Lambda^{\tau} / \operatorname{det} \Lambda$. The second row in Fig. 5 shows the results with directly transforming flow velocity as $\boldsymbol{v}_{f}^{\prime}=\Lambda \boldsymbol{v}_{f} / \operatorname{det} \Lambda$. Comparing the velocity distributions of two different methods, we can confirm the feasibility of controlling flow velocity with designed permeability.

\section{Discussion and conclusion}

We investigate conductive flux and radiation flux with the Fourier law and the Rosseland diffusion approximation, respectively. The Fourier law is reasonable at the macroscale, but it fails at the nanoscale where phonons are the carrier of heat transfer. ${ }^{39-42}$ The Rosseland diffusion approximation is valid in optically thick media, indicating that the mean free path of photons is far smaller than system size. Therefore, this approximation corresponds to a far-field effect. ${ }^{43}$ Although the present theory can deal with far-field thermal radiation, it cannot necessarily be used to handle near-field thermal radiation $^{44-53}$ which is governed by classic fluctuating electrodynamics theory. Therefore, the exploration on nearfield thermal radiation remains to be continued. For convective flux, we manipulate flow velocity with porous media which is dominated by the Darcy law. We also find that recent studies $^{54,55}$ handled drag-free flow with the Navier-Stokes equation, which can be an alternative method to deal with thermal convection.

Transformation theory does not restrict shapes, so these omnithermal devices can be extended to other complex shapes such as ellipses. ${ }^{16,17}$ Certainly, the present theory can also deal with any combination of the three basic modes of heat transfer as long as the transformation rules are satisfied. As experimental demonstrations, one may resort to the heat transfer in aerogels. ${ }^{5}$ The carrier of convective flux can be either fluids or gases in aerogels. We can also use multilayered structures ${ }^{10}$ to realize anisotropy and inhomogeneity. Other porous media with tunable porosity including ceramics, metals, and rocks are also potential candidates.

To sum up, we have established the theory of transformation omnithermotics which can control conduction, convection, and radiation simultaneously. In other words, a unification among the three basic modes of heat transfer is achieved within the theoretical framework of transformation omnithermotics. As model applications, we further design three devices including omnithermal cloaking, concentrating, and rotating, which are all confirmed by finite-element simulations. We also apply porous media to control flow velocity, ensuring the feasibility and completeness of this work. These results have potential applications in enhancing the efficiency of solar vapour generation or improving the performance of aerogel insulation, where the three basic modes of heat transfer must be taken into account simultaneously.

\section{Conflict of interest}

There are no conflicts to declare.

\section{Acknowledgements}

We acknowledge the financial support by the National Natural Science Foundation of China under Grant No. 11725521 .

\section{References}

1. H. Ghasemi, G. Ni, A. M. Marconnet, J. Loomis, S. Yerci, N. Miljkovic and G. Chen, Nat. Commun., 2014, 5, 4449.

2. K. Bae, G. Kang, S. K. Cho, W. Park, K. Kim and W. J. Padilla, Nat. Commun., 2015, 6, 10103.

3. F. Zhao, X. Y. Zhou, Y. Shi, X. Qian, M. Alexander, X. P. Zhao, S. Mendez, R. G. Yang, L. T. Qu and G. H. Yu, Nat. Nanotechnol., 2018, 13, 489-495. 
4. M. M. Gao, L. L. Zhu, C. K. Peh and G. W. Ho, Energ. Environ. Sci., 2019, 12, $841-864$.

5. X. Xu, Q. Q. Zhang, M. L. Hao, Y. Hu, Z. Y. Lin, L. L. Peng, T. Wang, X. X. Ren, C. Wang, Z. P. Zhao, C. Z. Wan, H. L. Fei, L. Wang, J. Zhu, H. T. Sun, W. L. Chen, T. Du, B. W. Deng, G. J. Cheng, I. Shakir, C. Dames, T. S. Fisher, X. Zhang, H. Li, Y. Huang and X. F. Duan, Science, 2019, 363, 723-727.

6. J. B. Pendry, D. Schurig and D. R. Smith, Science, 2006, 312, 1780-1782.

7. C. Z. Fan, Y. Gao and J. P. Huang, Appl. Phys. Lett., 2008, 92, 251907.

8. T. Y. Chen, C. N. Weng and J. S. Chen, Appl. Phys. Lett., 2008, 93, 114103.

9. J. P. Huang, ES Energy Environ., 2019, 6, 1-3.

10. S. Narayana and Y. Sato, Phys. Rev. Lett., 2012, 108, 214303.

11. H. Y. Xu, X. H. Shi, F. Gao, H. D. Sun and B. L. Zhang, Phys. Rev. Lett., 2014, 112, 054301.

12. T. C. Han, X. Bai, D. L. Gao, J. T. L. Thong, B. W. Li and C.W. Qiu, Phys. Rev. Lett., 2014, 112, 054302.

13. Y. G. Ma, Y. C. Liu, M. Raza, Y. D. Wang and S. L. He, Phys. Rev. Lett., 2014, 113, 205501.

14. T. C. Han, X. Bai, J. T. L. Thong, B. W. Li and C.W. Qiu, Adv. Mater., 2014, 26, 1731-1734.

15. Y. Li, X. Y. Shen, Z. H. Wu, J. Y. Huang, Y. X. Chen, Y. S. Ni and J. P. Huang, Phys. Rev. Lett., 2015, 115, 195503.

16. T. C. Han, P. Yang, Y. Li, D. Y. Lei, B. W. Li, K. Hippalgaonkar and C.W. Qiu, Adv. Mater., 2018, 30, 1804019.

17. J. Qin, W. Luo, P. Yang, B. Wang, T. Deng and T. C. Han, Int. J. Heat Mass Transfer, 2019, 141, 487-490.

18. J. X. Li, Y. Li, T. L. Li, W. Y. Wang, L. Q. Li and C.W. Qiu, Phys. Rev. Appl., 2019, 11, 044021.

19. T. C. Han, J. J. Zhao, T. Yuan, D. Y. Lei, B. W. Li and C.W. Qiu, Energ. Environ. Sci., 2013, 6, 3537-3541.

20. M. Moccia, G. Castaldi, S. Savo, Y. Sato and V. Galdi, Phys. Rev. X, 2014, 4, 021025.

21. R. S. Kapadia and P. R. Bandaru, Appl. Phys. Lett., 2014, 105, 233903.

22. T. Z. Yang, X. Bai, D. L. Gao, L. Z. Wu, B. W. Li, J. T. L. Thong and C.W. Qiu, Adv. Mater., 2015, 27, 7752-7758.

23. Y. Li, X. Bai, T. Z. Yang, H. Luo and C.W. Qiu, Nat. Commun., 2018, 9, 273.

24. R. Hu, S. L. Zhou, Y. Li, D. Y. Lei, X. B. Luo and C.W. Qiu, Adv. Mater., 2018, 30, 1707237.

25. S. L. Zhou, R. Hu and X. B. Luo,Int. J. Heat Mass Transfer, 2018, 127, 607613.

26. R. Hu, S. Y. Huang, M. Wang, X. L. Luo, J. Shiomi and C. W. Qiu, Adv. Mater., 2019, 31, 1807849.

27. X. Y. Peng and R. Hu, ES Energy Environ., 2019, 6, 39-44.

28. Z. Y. Zhou, X. Y. Shen, C. C. Fang and J. P. Huang, ES Energy Environ., 2019, 6, 85-91.
29. K. P. Vemuri and P. R. Bandaru, Appl. Phys. Lett., 2014, 104, 083901.

30. T. Z. Yang, K. P. Vemuri and P. R. Bandaru, Appl. Phys. Lett., 2014, 105, 083908.

31. K. P. Vemuri, F. M. Canbazoglu and P. R. Bandaru, Appl. Phys. Lett., 2014, 105, 193904.

32. G. L. Dai, J. Shang and J. P. Huang, Phys. Rev. E, 2018, 97, 022129.

33. Y. Li, K. J. Zhu, Y. G. Peng, W. Li, T. Z. Yang, H. X. Xu, H. Chen, X. F. Zhu, S. H. Fan and C. W. Qiu, Nat. Mater., 2019, 18, 48-54.

34. Y. Li, Y.G. Peng, L. Han, M. A. Miri, W. Li, M. Xiao, X. F. Zhu, J. L. Zhao, A. Alu, S. H. Fan and C.W. Qiu, Science, 2019, 364, 170-173.

35. F. B. Yang, L. J. Xu and J. P. Huang, ES Energy Environ., 2019, 6, 45-50.

36. L. J. Xu and J. P. Huang, Phys. Rev. Appl., 2019, 12, 044048.

37. http://www.comsol.com/

38. Y. A. Urzhumov and D. R. Smith, Phys. Rev. Lett., 2011, 107, 074501.

39. J. Ren, P. Hanggi and B. W. Li, Phys. Rev. Lett., 2010, 104, 170601.

40. J. Ren, S. Liu and B. W. Li, Phys. Rev. Lett., 2012, 108, 210603.

41. N. B. Li, J. Ren, L. Wang, G. Zhang, P. Hanggi and B. W. Li, Rer. Mod. Phys., 2012, 84, 1045-1066.

42. H. Bao, J. Chen, X. K. Gu and B. Y. Cao, ES Energy Environ., 2018, 1, 1655.

43. B. X. Wang, M. Q. Liu, T. C. Huang and C. Y. Zhao, ES Energy Environ., 2019, 6, 18-38.

44. P. Ben-Abdallah and S. A. Biehs, Phys. Rev. Lett., 2014, 112, 044301.

45. V. Kubytskyi, S. A. Biehs and P. Ben-Abdallah, Phys. Rev. Lett., 2014, 113, 074301.

46. S. A. Dyakov, J. Dai and M. Yan, Phys. Rev. B, 2014, 90, 045414.

47. S. A. Dyakov, J. Dai, M. Yan and M. Qiu, J. Phys. D: Appl. Phys., 2015, 48, 305104.

48. Z. Y. Li, EPL, 2015, 110, 14001

49. R. Messina, W. L. Jin and A. W. Rodriguez, Phys. Rev. B, 2016, 94, 205438.

50. V. Fernandez-Hurtado, F. J. Garcia-Vidal, S. F. Fan and J. C. Cuevas, Phys. Rev. Lett., 2017, 118, 203901.

51. M. Ghashami, H. Y. Geng, T. Kim, N. Iacopino, S. K. Cho and K. Park, Phys. Rev. Lett., 2018, 120, 175901.

52. S. C. Sun, ES Energy Environ., 2019, 6, 57-68.

53. H. Y. Yu, H. C. Zhang, Z. N. Dai and X. L. Xia, ES Energy Environ., 2019, 6, 69-77.

54. J. Park, J. R. Youn and Y. S. Song, Phys. Rev. Lett., 2019, 123, 074502.

55. J. Park, J. R. Youn and Y. S. Song, Phys. Rev. Appl., 2019, 12, 061002.

Publisher's Note Engineered Science Publisher remains neutral with regard to jurisdictional claims in published maps and institutional affiliations. 